\title{
Early Development of Bidirectional Associations between Sleep Disturbance and Diabetes
}

\author{
Yongin Cho \\ Division of Endocrinology and Metabolism, Department of Internal Medicine, Inha University School of Medicine, Incheon, Korea
}

Sleep accounts for one-third to one-fourth of a human's lifetime. Proper sleep is crucial for maintaining general and metabolic health. Sleep disturbances include all disorders of initiating and maintaining sleep and are inherently associated with an increase in various noncommunicable diseases. Qualitative or quantitative disturbances during sleep increase the prevalence of obesity, diabetes, cardiovascular diseases, and hypertension [1]. Over the past decade, the prevalence rates of sleep disturbances and type 2 diabetes mellitus (T2DM) have been increasing dramatically worldwide. The association between sleep disorders and diabetes is bidirectional because chronic sleep disorders increase the risk of insulin resistance/diabetes, and diabetes worsens the quality of sleep [2].

Numerous community-based and hospital-based epidemiological studies suggest that both quantitative and qualitative disorders of sleep significantly increase the risk of diabetes. Regarding quantity, both prolonged and short durations of sleep increase the risk of diabetes. Yaggi et al. [3] reported that people who sleep for less than 5 hours/night have two times higher risk of developing diabetes, and those who sleep for more than 8 hours have three times higher risk than those who sleep for 7 hours a day. According to a meta-analysis assessing the doseresponse relationship between duration of sleep and risk of T2DM, the lowest risk of T2DM was observed in individuals who slept 7 to 8 hours a day. Shorter and longer durations of sleep were associated with a higher risk of T2DM [4]. These quantitative disorders of sleep have been associated with increased insulin resistance, upregulation of appetite, and reduced energy expenditure $[5,6]$. Not only the quantity of sleep, but also the quality of sleep is closely associated with development of diabetes. In a recent meta-analysis, poor sleep quality was associated with increased glycosylated hemoglobin (HbA1c) (weighted mean difference, $0.35 \%$; $95 \%$ confidence interval, 0.12 to 0.58 ) in patients with T2DM [7]. Obstructive sleep apnea, a representative disease that lowers the quality of sleep, is characterized by chronic intermittent hypoxia and increased sympathetic activity. It increases insulin resistance by altering the metabolism of glucose and lipids and the levels of stress hormones and appetite-related hormones [8]. Sleep disturbance can also indirectly affect glycemic control by disrupting diabetes self-management [9].

Conversely, frequent nocturia, nocturnal hypoglycemia, restless leg syndrome, and depression, which are relatively common in patients with diabetes, cause frequent nocturnal awakening and deteriorate sleep quality [10]. Peripheral neuropathy and related symptoms are also associated with sleep disturbances [11]. This reverse causality is supported by findings from studies conducted in non-obese patients with type 1 diabetes who had a high prevalence of obstructive sleep apnea $[12,13]$. Diabetic neuropathy affects central control of respiration and the upper airway neural reflex, facilitating sleep-disordered breathing. Furthermore, sleep-disordered breathing causes intermittent hypoxemia and hypercapnia, increases oxidative stress, and results in inflammation and sleep disturbance $[7,14]$.

In the article titled "Deterioration of sleep quality according
Corresponding author: Yongin Cho (D) https://orcid.org/0000-0002-4645-816X Division of Endocrinology and Metabolism, Department of Internal Medicine, Inha University School of Medicine, 27 Inhang-ro, Jung-gu, Incheon 22332, Korea E-mail: choyorin@gmail.com
This is an Open Access article distributed under the terms of the Creative Commons Attribution Non-Commercial License (https://creativecommons.org/licenses/by-nc/4.0/) which permits unrestricted non-commercial use, distribution, and reproduction in any medium, provided the original work is properly cited. 
to glycemic status," Hur et al. [15] investigated the effect of glycemic status on sleep quality in individuals with T2DM, prediabetes, and normal glucose tolerance. To evaluate the prevalence of sleep disorders in patients with prediabetes or early diabetes, the present study recruited patients without overt symptomatic neuropathy. Objective sleep quality was measured with the actigraph wrist-worn device (Actigraph Corp., Pensacola, FL, USA), and sleep efficiency decreased across normal glucose tolerance, prediabetes, and T2DM groups $(90.22 \%, 87.99 \%$, and $86.25 \%$, respectively; $P<0.05)$. Although the participants were not necessarily conscious of their sleep disturbances, their $\mathrm{HbA1c}$ level revealed a significant negative correlation with sleep efficiency $(r=-0.348, P=0.001)$.

Cho et al. [16] reported the associations between snoring in prediabetes and diabetes in a Korean population. Snoring is the most common and prominent symptom of obstructive sleep apnea that inhibits good-quality sleep. Data of self-reported multiple characteristics of snoring were collected from 3,948 middle-aged adults without prior cardiovascular diseases. Participants with the most severe snoring were at 1.84 times higher risk for prediabetes and 2.24 times higher risk for diabetes than non-snorers. Such graded association was also observed among the most frequent snorers with higher risk for prediabetes (odds ratio [OR], 1.78) and diabetes (OR, 2.03; all $P<0.05)$. Higher snoring intensity and higher frequency were positively associated with fasting glucose and $\mathrm{HbAlc}$ levels, and the association remained robust after additional adjustment for sleep duration, excessive daytime sleepiness, unwakefulness, and sleep-deprived driving. As such, the association between sleep disturbances and dysglycemia is significant and begins even before the development of T2DM. Deterioration of sleep quality was confirmed in patients with prediabetes, and low sleep quality through snoring was related to elevated blood glucose level in otherwise metabolically healthy adults.

It is not yet clear whether sleep quality deteriorates even in the pre-diabetes stages; however, related research results have been proposed recently. Engeda et al. [17] reported that clinically identified pre-diabetes was associated with poor sleep quality as assessed using a questionnaire on trouble maintaining sleep, waking up too early, and short sleep. Some of the complications of diabetes, such as peripheral neuropathy or autonomic neuropathy, which are associated with poor sleep quality [18], have been reported not only in patients with a long duration of diabetes, but also in patients with prediabetes or early T2DM [19]. In the above study, the prevalence rate of peripheral neuropathy was $49 \%$ in adults with prediabetes and $50 \%$ in patients with new-onset diabetes. These findings suggest that early diagnosis and management of sleep disturbances are required in patients with prediabetes or early T2DM. The prevalence of sleep apnea syndrome was confirmed to increase with progression of pre-diabetes. Furthermore, evidence suggests that obstructive sleep apnea can alter glucose metabolism in individuals without T2DM [20,21]. Treatment of obstructive sleep apnea using continuous positive airway pressure, which is the gold standard treatment for obstructive sleep apnea, significantly improved insulin sensitivity and glucose metabolism both in patients without diabetes [22] and in those with prediabetes [23].

As noted above, diabetes and sleep disturbance are closely associated with each other, and this bidirectional association seems to develop before onset of T2DM. Uncontrolled diabetes itself causes sleep disturbances, and sleep disturbances induce high blood glucose, which can lead to vicious cycles of diabetes and sleep disturbances. Furthermore, as the prevalence of diabetes and sleep disturbances increase, more attention should be paid to the importance of their relationship. However, in clinical practice, efforts to evaluate the quality and amount of sleep in patients with diabetes are insufficient. Proper diet, exercise, and weight control are important to consider when physicians assess and educate patients with diabetes. Similarly, sleep patterns should be regarded as one of the important alterable factors of lifestyle modifications in patients with diabetes. Likewise, the need to assess diabetes risk should be emphasized in patients with sleep disturbances. Appropriate assessment and therapeutic approach can help these patients improve glucose control and sleep disturbances.

\section{CONFLICTS OF INTEREST}

No potential conflict of interest relevant to this article was reported.

\section{ACKNOWLEDGMENTS}

This work was supported by an Inha University Hospital research grant.

\section{REFERENCES}

1. Buxton OM, Marcelli E. Short and long sleep are positively as- 
sociated with obesity, diabetes, hypertension, and cardiovascular disease among adults in the United States. Soc Sci Med 2010;71:1027-36.

2. Chattu VK, Chattu SK, Burman D, Spence DW, Pandi-Perumal SR. The interlinked rising epidemic of insufficient sleep and diabetes mellitus. Healthcare (Basel) 2019;7:37.

3. Yaggi HK, Araujo AB, McKinlay JB. Sleep duration as a risk factor for the development of type 2 diabetes. Diabetes Care 2006;29:657-61.

4. Shan Z, Ma H, Xie M, Yan P, Guo Y, Bao W, Rong Y, Jackson CL, Hu FB, Liu L. Sleep duration and risk of type 2 diabetes: a meta-analysis of prospective studies. Diabetes Care 2015;38: 529-37.

5. St-Onge MP, O'Keeffe M, Roberts AL, RoyChoudhury A, Laferrere B. Short sleep duration, glucose dysregulation and hormonal regulation of appetite in men and women. Sleep 2012; 35:1503-10.

6. St-Onge MP. The role of sleep duration in the regulation of energy balance: effects on energy intakes and expenditure. J Clin Sleep Med 2013;9:73-80.

7. Lee SWH, Ng KY, Chin WK. The impact of sleep amount and sleep quality on glycemic control in type 2 diabetes: a systematic review and meta-analysis. Sleep Med Rev 2017;31:91-101.

8. Knutson KL. Impact of sleep and sleep loss on glucose homeostasis and appetite regulation. Sleep Med Clin 2007;2:187-97.

9. Chasens ER, Korytkowski M, Sereika SM, Burke LE. Effect of poor sleep quality and excessive daytime sleepiness on factors associated with diabetes self-management. Diabetes Educ 2013;39:74-82.

10. Surani S, Brito V, Surani A, Ghamande S. Effect of diabetes mellitus on sleep quality. World J Diabetes 2015;6:868-73.

11. Neumann C, Martinez D, Schmid H. Nocturnal oxygen desaturation in diabetic patients with severe autonomic neuropathy. Diabetes Res Clin Pract 1995;28:97-102.

12. Janovsky CC, Rolim LC, de Sa JR, Poyares D, Tufik S, Silva AB, Dib SA. Cardiovascular autonomic neuropathy contributes to sleep apnea in young and lean type 1 diabetes mellitus patients. Front Endocrinol (Lausanne) 2014;5:119.

13. Banghoej AM, Nerild HH, Kristensen PL, Pedersen-Bjergaard U, Fleischer J, Jensen AE, Laub M, Thorsteinsson B, Tarnow L. Obstructive sleep apnoea is frequent in patients with type 1 diabetes. J Diabetes Complications 2017;31:156-61.
14. Ficker JH, Dertinger SH, Siegfried W, Konig HJ, Pentz M, Sailer D, Katalinic A, Hahn EG. Obstructive sleep apnoea and diabetes mellitus: the role of cardiovascular autonomic neuropathy. Eur Respir J 1998;11:14-9.

15. Hur MH, Lee MK, Seong K, Hong JH. Deterioration of sleep quality according to glycemic status. Diabetes Metab J 2020;44: 679-86.

16. Cho SMJ, Lee H, Shim JS, Kim HC. Association of snoring with prediabetes and type 2 diabetes mellitus: the cardiovascular and metabolic diseases etiology research center cohort. Diabetes Metab J 2020;44:687-98.

17. Engeda J, Mezuk B, Ratliff S, Ning Y. Association between duration and quality of sleep and the risk of pre-diabetes: evidence from NHANES. Diabet Med 2013;30:676-80.

18. Mallien J, Isenmann S, Mrazek A, Haensch CA. Sleep disturbances and autonomic dysfunction in patients with postural orthostatic tachycardia syndrome. Front Neurol 2014;5:118.

19. Lee CC, Perkins BA, Kayaniyil S, Harris SB, Retnakaran R, Gerstein HC, Zinman B, Hanley AJ. Peripheral neuropathy and nerve dysfunction in individuals at high risk for type 2 diabetes: the PROMISE cohort. Diabetes Care 2015;38:793-800.

20. Seicean S, Kirchner HL, Gottlieb DJ, Punjabi NM, Resnick H, Sanders M, Budhiraja R, Singer M, Redline S. Sleep-disordered breathing and impaired glucose metabolism in normal-weight and overweight/obese individuals: the Sleep Heart Health Study. Diabetes Care 2008;31:1001-6.

21. Kent BD, Grote L, Bonsignore MR, Saaresranta T, Verbraecken J, Levy P, Sliwinski P, Tkacova R, Kvamme JA, Fietze I, Hedner J, McNicholas WT; European Sleep Apnoea Database collaborators. Sleep apnoea severity independently predicts glycaemic health in nondiabetic subjects: the ESADA study. Eur Respir J 2014;44:130-9.

22. Salord N, Fortuna AM, Monasterio C, Gasa M, Perez A, Bonsignore MR, Vilarrasa N, Montserrat JM, Mayos M. A randomized controlled trial of continuous positive airway pressure on glucose tolerance in obese patients with obstructive sleep apnea. Sleep 2016;39:35-41.

23. Weinstock TG, Wang X, Rueschman M, Ismail-Beigi F, Aylor J, Babineau DC, Mehra R, Redline S. A controlled trial of CPAP therapy on metabolic control in individuals with impaired glucose tolerance and sleep apnea. Sleep 2012;35:617-25B. 